\title{
Effect of Interfering Users on the Modulation Order and Code Rate for UWB Impulse-Radio Bit-Interleaved Coded M-ary PPM
}

\author{
Ruben Merz and Jean-Yves Le Boudec \\ EPFL, School of Computer and Communication Sciences \\ CH-1015 Lausanne, Switzerland \\ phone: (+41) 21693 6616, fax: (+41) 216936610 \\ \{ruben.merz, jean-yves. leboudec\} depfl.ch
}

\begin{abstract}
-
We consider the impact of multi-user interference on a bitinterleaved coded-modulation system with M-ary PPM (BIC Mary PPM) in an impulse-radio ultra-wideband physical layer. In a realistic scenario such as an ad hoc network, the interference is inherently variable. This justifies the need for a physical layer that can optimally adapt its transmission parameters to the interference level. We use puncturing on the channel code so that we can not only change the modulation order $M$ but also the channel code rate. We study by simulation how the optimal combination of modulation order $M$ and channel code rate behaves with various degrees of interference. The results show that BIC M-ary PPM can be successfully adapted to various levels of interference conditions. It also shows the benefit of both rate and modulation adaptation, especially in the presence of multi-user interference.
\end{abstract}

\section{INTRODUCTION}

Recent results [1], [2] in the field of medium-access control (MAC) for UWB time-hopping impulse-radio ad-hoc networks have demonstrated the advantages of rate adaptation. With rate adaptation, nodes do not attempt to control the interference they create for concurrent users. Instead, they adapt their transmission rate to the current level of interference. In [1] and [2] a transmitter adapts its rate by dynamically changing the channel code rate based on feedback from its destination. This scheme is very efficient in the presence of a high level of interference. However, when the network is lightly loaded, the possibility of augmenting the modulation order would permit for a great increase in spectral efficiency.

This motivates us to study the performance of a physical layer that can not only adapt its channel code rate, but also increase the modulation order. We selected bit-interleaved coded-modulation (BICM) [3]. It has a low complexity and can be easily used with any type of binary channel code. We use it with M-ary pulse position modulation (M-ary PPM).

In this paper, we are not interested in optimizing the channel code or interleaver design. Rather, we want to study

The work presented in this paper was supported (in part) by the National Competence Center in Research on Mobile Information and Communication Systems (NCCR-MICS), a center supported by the Swiss National Science Foundation under grant number 5005-67322, and by CTI contract No7109.2;1 ESPP-ES the interaction between the modulation order $M$ and the channel code rate $R_{c}$. Furthermore, we want to show how this relatively simple design can yield to substantial performance improvements.

Bit-interleaved coded modulation (BICM) was proposed in [3] and [4] and M-ary PPM for UWB was analyzed in [5]. Previous work on combined modulation and channel coding can be found in [6], [7], [8] and [9]; they all consider signal constellations in two dimensional spaces. Hence, they do not take advantage of the infrequent pulse transmission characteristics of pulse based physical layers. Instead of changing the modulation order to increase the spectral efficiency, it is possible to make the pulse transmission period (PTP) variable as in [10] and [11]. However, makes the average transmitted power on the channel variable if the power used to send a single pulse is fixed. Note that [10] considers M-ary PPM but without channel coding and multi-user interference (MUI).

Since we consider the effect of multi-user interference, we cannot use the Gaussian approximation on the interference [12]. Hence, we analyze by simulation the performance of the BIC M-ary PPM physical layer. We consider a multipath channel and several concurrent transmitters. We do not consider narrowband interference. We show the packet error rate (PER) in various topologies and interference conditions. We also analyze the best combination $\left(M, R_{c}\right)$ of modulation order $M$ and channel code rate $R_{c}$ in the presence of multiuser interference.

\section{SySTEM MODEL AND ASSUMPTIONS}

We consider a bit-interleaved coded-modulation system (BICM) as described in [3]. We use M-ary pulse position modulation (PPM) with time-hopping [5] and a binary ratecompatible punctured convolutional (RCPC) code of rate $R_{c}$ [13]. We assume a multipath propagation channel and a coherent, single user, Rake receiver. From the channel encoder and the modulator, BICM is obtained by concatenating the output of the convolutional encoder with the M-ary PPM modulator through a bit interleaver $\Pi$. In this paper, we assume a perfect random interleaver. Following the terminology of [3], we denote with $\mathcal{X}=\{0,1, \ldots, M-1\}$ the so called signal 
set of size $|\mathcal{X}|=M=2^{k}$. We also define the binary labeling map

$$
\mu_{M}:\left\{c_{1}, \ldots, c_{k}\right\} \in\{0,1\}^{k} \rightarrow x \in \mathcal{X}
$$

The binary labeling map $\mu$ models the modulator. In the case of M-ary PPM, the set $\mathcal{X}$ corresponds to the set of available pulse positions.

The convolutional encoder produces coded blocks $\boldsymbol{c}=$ $\left[c_{0}, c_{2}, \ldots, c_{k * K-1}\right]$ of $k * K$ coded bits. Each coded block $\boldsymbol{c}$ is interleaved. The output $\Pi(\boldsymbol{c})$ of the interleaver is broken into sub-blocks of $k$ bits. Then, these sub-blocks are mapped into one of the $M$ signals in $\mathcal{X}$. An illustration of the transmitter and receiver chain is given in Figure 1.

From the modulator, the signal transmitted by the $i^{t h}$ source is

$$
s^{(i)}(t)=\sum_{j=0}^{K-1} p\left(t-j T_{f}-c_{j}^{(i)} T_{c}-x_{j}^{(i)} T_{m}\right)
$$

where $\left\{x_{j}^{(i)}\right\}_{j=0}^{K-1}$ is the symbol sequence with $x_{j}^{(i)} \in \mathcal{X}, K$ is the block (or packet) length $T_{c}$ is the chip width, $T_{f}$ is the frame length, $T_{m}$ is the pulse position offset and $c_{j}^{(i)}$ is the Time-Hopping Sequence (THS). The THS is a sequence of integers uniformly distributed in $[0, P T P-(M+1)]$ where $P T P=\frac{T_{f}}{T_{c}}$ is the Pulse Transmission Period. We assume that there is no inter-symbol interference or intra-symbol interference (ISI). The constraint on the inter-symbol interference can be enforced by having a guard time $T_{g}$ at the end of each frame, or by constraining the THS such that the minimum spacing between two consecutive chips is larger than $T_{g}$. The constraint on the intra-symbol interference can be enforced by choosing a sufficiently large $T_{m}$. The pulse $p(t)$ has unit energy i.e. $\int|p(t)|^{2} d t=1$.

The channel impulse response between the $i^{t h}$ transmitter and the receiver is

$$
h^{(i)}(t)=\sum_{l=0}^{L-1} \alpha_{l}^{(i)} \delta\left(t-\nu_{l}^{(i)}\right)
$$

where $\delta$ is a Dirac function, $\nu_{l}^{(i)}$ is the delay induced by the $l^{\text {th }}$ path and $L$ the maximum number of paths. We denote by $A^{(i)}=\sum_{l=0}^{L-1}\left(\alpha_{l}^{(i)}\right)^{2}$ the total energy of the channel. The channel is considered to be static for the duration of a packet transmission.

At the receiver side, we consider a coherent, single user, Rake receiver. However, the number of branches of the Rake receiver is assumed to be limited to $\tilde{L} \leq L$. The received signal is

$$
r(t)=\sum_{i=1}^{U} h^{(i)}(t) * s^{(i)}\left(t-\phi^{(i)}\right)+n(t)
$$

where $*$ is the convolution operator, $U$ is the number of transmitters present in the system, $\phi^{(i)} \in\left[0, T_{f}\right)$ is the delay between the $i^{t h}$ transmitter and the receiver, and $n(t)$ is zero mean white Gaussian noise with two-sided power spectral density $\frac{N_{0}}{2}$.

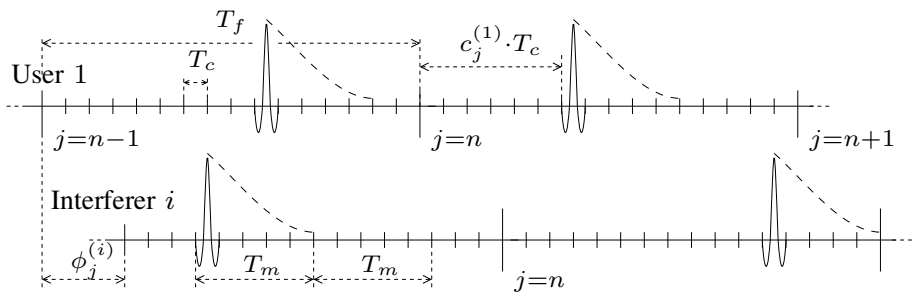

Fig. 2. Illustration of the definitions. $\phi^{(i)}$ is the delay between interferer $i$ and the source, $T_{f}$ is the frame length, $T_{c}$ is the chip width and $T_{m}$ is the pulse position offset. The dashed curve following the pulses represents the multipath propagation. Note that the pulse position offset $T_{m}$ is not necessary equal to the chip width $T_{c}$.

The autocorrelation $\Theta(\tau)$ of the pulse $p(t)$ is $\Theta(\tau)=$ $\int_{-\infty}^{\infty} p(t) p(t-\tau) d t$ The pulse shape of $p(t)$ at the receiver is the second derivative of a Gaussian pulse, i.e.

$$
p(t)=C_{E}\left(1-4 \pi\left(\frac{t}{\tau_{p}}\right)^{2}\right) \exp \left(-2 \pi\left(\frac{t}{\tau_{p}}\right)^{2}\right)
$$

where $\tau_{p}$ is a time normalization factor and $C_{E}$ an energy normalization factor. Hence, with (5), the autocorrelation $\Theta(\tau)$ is

$$
\Theta(\tau)=\left[1-4 \pi\left(\frac{\tau}{\tau_{p}}\right)^{2}+\frac{4 \pi^{2}}{3}\left(\frac{\tau}{\tau_{p}}\right)^{4}\right] \exp \left[-\pi\left(\frac{\tau}{\tau_{p}}\right)^{2}\right]
$$

If $T_{p}$ is the width of the pulse, the autocorrelation $\Theta(\tau)$ is equal to zero outside the interval $\left[-T_{p}, T_{p}\right]$.

\section{BIT-INTERLEAVED CODED M-ARY PPM}

In this section, we first compute the outputs of the matched filters of the M-ary PPM demodulator. We then show how these outputs are used in the decision rule of [3] for the channel decoder.

\section{A. Output of the M-ary PPM matched filters}

We let $i=1$ be the user of interest. We assume perfect channel knowledge and perfect synchronization between the transmitter 1 and the receiver i.e. $\phi_{1}=0$. Since we have $M$ matched filter outputs, we have $M$ template waveform $w_{m}(t)$, $m=0,1, \ldots, M-1$, matched on the signal from the first transmitter where

$$
\begin{aligned}
& w_{m}(t)=\sum_{j=0}^{K-1} h^{(1)}(t) * p\left(t-j T_{f}-c_{j}^{(1)} T_{c}-m T_{m}\right) \\
= & \sum_{j=0}^{K-1} \sum_{l=0}^{\tilde{L}-1} \alpha_{l}^{(1)} \cdot p\left(t-j T_{f}-c_{j}^{(1)} T_{c}-m T_{m}-\nu_{l}^{(1)}\right)
\end{aligned}
$$

Then, the $j^{t h}$ output $r_{j, m}$ of the $m^{t h}$ matched filter is

$$
r_{j, m}=\int_{j T_{f}}^{(j+1) T_{f}} r(t) w_{m}(t) d t
$$




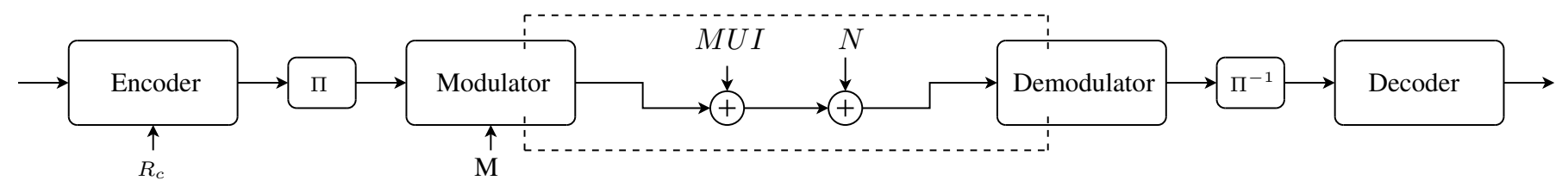

Fig. 1. Block diagram of the BIC MPPM physical layer. The bit interleaver is denoted by $\Pi, R_{c}$ denotes the code rate and $M$ is the modulation order. The channel, where multi-user interference (MUI) and noise $(N)$ is added, is depicted with the dashed box.

Using equations (2),(4) and (6), the right-hand side of (7) becomes

$$
\begin{aligned}
r_{j, m}= & \sum_{i=1}^{U} \sum_{l=0}^{L-1} \alpha_{l}^{(i)} \sum_{\tilde{l}=0}^{\tilde{L}-1} \alpha_{\tilde{l}}^{(1)} \int_{j T_{f}}^{(j+1) T_{f}} \\
& p\left(t-j T_{f}-c_{j}^{(i)} T_{c}-x_{j}^{(i)} T_{m}-\phi^{(i)}-\nu_{l}^{(i)}\right) \\
& p\left(t-j T_{f}-c_{j}^{(1)} T_{c}-m T_{m}-\nu_{\tilde{l}}^{(1)}\right) d t
\end{aligned}
$$

After a few manipulations, the previous expression can be rewritten as

$$
r_{j, m}=s_{j, m}+\sum_{i=2}^{I} I_{j, m}^{(i)}+n_{j, m}
$$

The contribution $s_{j, m}$ of the user of interest at the output of the $m^{t h}$ matched filter is

$$
\begin{aligned}
s_{j, m} & =\sum_{l=0}^{\tilde{L}-1}\left(\alpha_{l}^{(1)}\right)^{2} \cdot \Theta\left[\left(m-x_{j}^{(1)}\right) T_{m}\right] \\
& =\sum_{l=0}^{\tilde{L}-1}\left(\alpha_{l}^{(1)}\right)^{2} 1_{\left\{x_{j}^{(1)}=m\right\}}
\end{aligned}
$$

In a similar way, we can obtain the contribution of the $i^{t h}$, $i>1$, interferer is

$$
I_{j, m}^{(i)}=\sum_{\tilde{l}=0}^{\tilde{L}-1} \alpha_{\tilde{l}}^{(1)} \sum_{l=0}^{L-1} \alpha_{l}^{(i)} \Theta\left[\Delta_{j, m}^{(i)}+\left(\nu_{\tilde{l}}^{(1)}-\nu_{l}^{(i)}\right)-\phi^{(i)}\right]
$$

where (see Figure 2)

$\Delta_{j, m}^{(i)}=\left\{\begin{array}{l}\left(c_{j}^{(1)}-c_{j}^{(i)}\right)+\left(m-x_{j}^{(i)}\right) T_{m}, \quad \text { if } c_{j}^{(1)} \cdot T_{c}>\phi^{(i)} \\ \left(c_{j}^{(1)}-c_{j-1}^{(i)}\right)+\left(m-x_{j-1}^{(i)}\right) T_{m}-T_{f}, \text { otherwise }\end{array}\right.$

Finally, the filtered white noise $n_{j, m} \sim \mathcal{N}\left(0, \sigma_{N}^{2}\right)$ with $\sigma_{N}^{2}=\frac{N_{0}}{2}$

With equation (8), the original continuous time channel of equation (4) is transformed into $M$ parallel discrete time channels. In other words, the continuous time channel is mapped with an equivalent vector channel $p(\boldsymbol{y} \mid \boldsymbol{x})$ of dimension $M$.

\section{B. BIC M-ary PPM decoder}

We first recall the results from [3] and then describe how they are used in our case. We define $\mathcal{X}_{b}^{i}$ as the subset of all signals $\boldsymbol{x} \in \mathcal{X}$ whose label has the value $b \in\{0,1\}$ in position $i$. For instance, with $k=3$, the subset $\mathcal{X}_{2}^{1}$ is
$\{010,011,110,111\}$. The channel decoder makes decisions according to the maximum likelihood (ML) rule

$$
\hat{\boldsymbol{c}}=\arg \max _{\mathrm{c} \in \mathcal{C}} \sum_{j} \lambda^{i}\left(\boldsymbol{y}_{j}, c_{j}\right)
$$

for $i=1, \ldots, k$ where for $b \in\{0,1\}$

$$
\lambda^{i}\left(\boldsymbol{y}_{j}, b\right)=\log \sum_{\boldsymbol{x} \in \mathcal{X}_{b}^{i}} p\left(\boldsymbol{y}_{j} \mid \boldsymbol{x}\right)
$$

is the ML bit metric. The decision rule (13) is easily implemented with a Viterbi decoder. This is a very attractive feature from an implementation point of view.

For $M>2$, the decision metric in (13) can be computationally too complex. Also, it necessitates the knowledge of $\sigma_{N}^{2}$. Hence for $M>2$, the $\log$-sum approximation $\log \sum_{j} z_{j} \simeq$ $\max _{j} \log z_{j}$ (see [4]) can be used to obtain the following simplified bit metrics

$$
\tilde{\lambda}^{i}\left(\boldsymbol{y}_{j}, b\right)=\max _{\boldsymbol{x} \in \mathcal{X}_{b}^{i}} \log p\left(\boldsymbol{y}_{j} \mid \boldsymbol{x}\right)
$$

Example 1: With $M=4$, the binary labeling map $\mu$ is

$$
\mu:\{00,01,11,10\} \rightarrow\left\{x_{1}, x_{2}, x_{3}, x_{4}\right\}
$$

Hence, for $i=1$ and $b=0$, the bit metrics (14) and (15) are

$$
\lambda^{1}\left(\boldsymbol{y}_{j}, 0\right)=\log \left[p\left(\boldsymbol{y}_{j} \mid x_{1}\right)+p\left(\boldsymbol{y}_{j} \mid x_{2}\right)\right]
$$

and

$$
\tilde{\lambda}^{1}\left(\boldsymbol{y}_{j}, 0\right)=\max \left[\log p\left(\boldsymbol{y}_{j} \mid x_{1}\right), \log p\left(\boldsymbol{y}_{j} \mid x_{2}\right)\right]
$$

Now in the case of a single user receiver, we can express the suboptimal bit metric (18) as a function of the matched filter outputs. It is straightforward to show that

$$
\tilde{\lambda}^{1}\left(\boldsymbol{y}_{j}, 0\right)=\max \left[r_{j, 1}, r_{j, 2}\right]
$$

Similarly,

$$
\begin{aligned}
& \tilde{\lambda}^{1}\left(\boldsymbol{y}_{j}, 1\right)=\max \left[r_{j, 3}, r_{j, 4}\right] \\
& \tilde{\lambda}^{2}\left(\boldsymbol{y}_{j}, 0\right)=\max \left[r_{j, 1}, r_{j, 4}\right] \\
& \tilde{\lambda}^{2}\left(\boldsymbol{y}_{j}, 1\right)=\max \left[r_{j, 2}, r_{j, 3}\right]
\end{aligned}
$$




\begin{tabular}{|c|c|c|c|}
\hline$T_{f}$ & $T_{c}$ & $T_{m}$ & $K$ \\
\hline $500 \mathrm{~ns}$ & $1 \mathrm{~ns}$ & $50 \mathrm{~ns}$ & 1024 bits \\
\hline \hline$\tilde{L}$ & $\tau_{p}$ & $E_{p}$ & $N_{0}$ \\
\hline 4 & 0.2877 & $0.2818 \mathrm{~mW}$ & $-80 \mathrm{dBm}$ \\
\hline
\end{tabular}

TABLE I

NUMERICAL VALUES OF THE PARAMETERS OF THE PHYSICAL LAYER

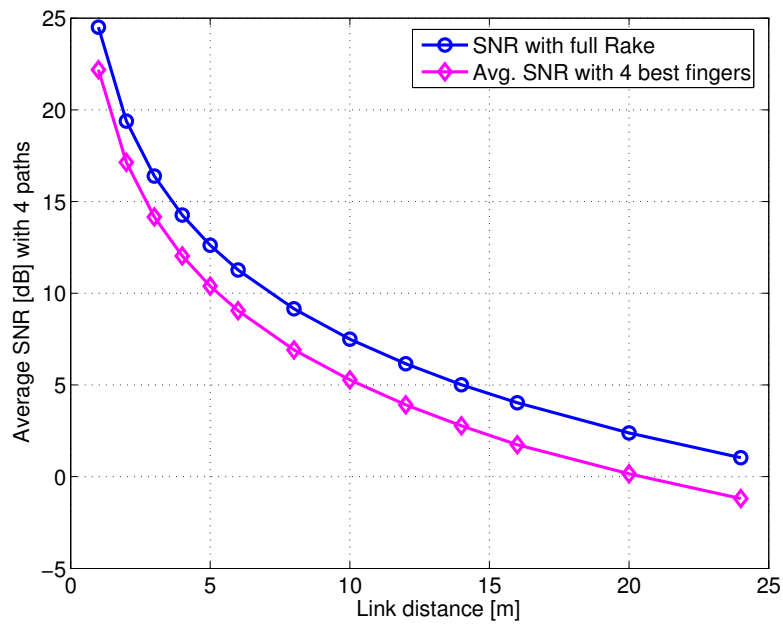

Fig. 3. Comparison of the average SNR obtained with a 4 fingers Rake receiver and the theoretical maximum SNR. The maximum SNR could be obtained with a full Rake receiver. This figure also provides the correspondence between the link distance and the SNR.

\section{PERFormance EVAluation}

In this section, we evaluate the performance of the BIC Mary PPM physical layer in various conditions, most notably in the presence of multi-user interference. Our two main performance metrics are the rate and the packet error rate (PER). The numerical values of the parameters of the physical layer are given in Table I. We do not use a perfect Rake receiver. Instead, we use a selective Rake receiver (S-Rake) [14] with $\tilde{L}=4$. With $\tilde{L}=4$, the performance loss is around 2 or $3 \mathrm{~dB}$ in terms of SNR [14]. This is confirmed by simulation; on Figure 3, we display the average SNR with respect to the theoretical SNR that would be obtained with a full Rake receiver. Even tough we gather only $\tilde{L}$ paths of the channel impulse response, a large enough value of $T_{m}$ is necessary in order to accommodate for the delay spread of the multipath channel. This ensures the orthogonality of the modulation.

We selected the RCPC codes from [15]; the mother code has rate $\frac{1}{4}$. Along with the uncoded case $\left(R_{c}=1\right)$, they provide the following set of available rates

$$
\begin{aligned}
\mathcal{R} & =\left\{R_{0}, R_{1}, \ldots, R_{24}\right\} \\
& =\{1,8 / 9,8 / 10,8 / 11, \ldots, 8 / 32\}
\end{aligned}
$$

of size $|\mathcal{R}|=25$. The block (or packet length) is $K=1024$ bits.

We consider $M=2,4,8$. The maximum value of the modulation order is limited by the frame length $T_{f}$. Following [3], we use a Gray mapping for the modulation map $\mu$. For $M=4$

$$
\mu_{4}:\{00,01,11,10\} \rightarrow\{0,1,2,3\}
$$

and for $M=8$

$$
\begin{gathered}
\mu_{8}:\{000,001,011,010,110,111,101,100\} \\
\rightarrow\{0,1,2,3,4,5,6,7\}
\end{gathered}
$$

The channel parameters are chosen according to the IEEE 802.15.4a CM1 channel model [16]. The path loss attenuation is computed according to [17]. All the simulations have been performed using Matlab. We consider three interference scenarios. A first scenario with no multi-user interference $(U=1)$, a second scenario with one interferer at one meter from the receiver $(U=2)$ and a third scenario with five interferers at one meter from the receiver $(U=6)$. We present the results as a function of the link distance between the transmitter of interest and the receiver. The correspondence between the average received SNR and the link distance is given by Figure 3 .

\section{A. Effect of the Multi-User Interference on the Packet Error Rate}

On Figure 4(a) we show the PER with no MUI for $R_{c}=\frac{4}{5}$, $R_{c}=\frac{1}{2}$ and $R_{c}=\frac{1}{4}$ for $M=2,4,8$. Similarly, on Figure 4(b) we show the PER for for the same set of modulation order and channel code rate in the presence of five interferers. On Figure 5(a) and Figure 5(b), we fix $M=2$ and compare the PER with $R_{c}=1$ (uncoded), $R_{c}=\frac{4}{5}$ and $R_{c}=\frac{1}{2}$. Already with $R_{c}=\frac{4}{5}$ we can observe a significant decrease of the PER with respect to the uncoded case.

A traditional way to compute the PER if only the bit error rate (BER) is available is to use

$$
\widehat{P E R}=1-(1-B E R)^{K}
$$

This assumes that all the bits of the packet are independent and identically distributed (i.i.d). Since we can also obtain the BER from our simulations, we compare (21) with the PER we obtained by simulations. As can be observed on Figure 4 with $M=2$, equation (21) is only valid in the uncoded case without MUI. In the other cases, using (21) always overestimates the PER. Results are similar with $M=4,8$. Indeed, the MUI breaks the i.i.d assumption.

\section{B. Effect of the Multi-User Interference on the Best Combi- nation of Modulation and Channel Code}

For each of the scenarios, we computed for each link distance the PER for each modulation order and a subset of the available channel code rate ${ }^{1}$. Then for each modulation order, we looked for the best rate, i.e. the maximum channel code rate assuming a maximum PER of $1 \%$. From these curves, we can infer the best combination of modulation order and channel code rate. The results are presented on Figure 6 and the best

${ }^{1}$ Every two channel codes starting from $R_{c}=1$. Hence there are 13 available rates. 


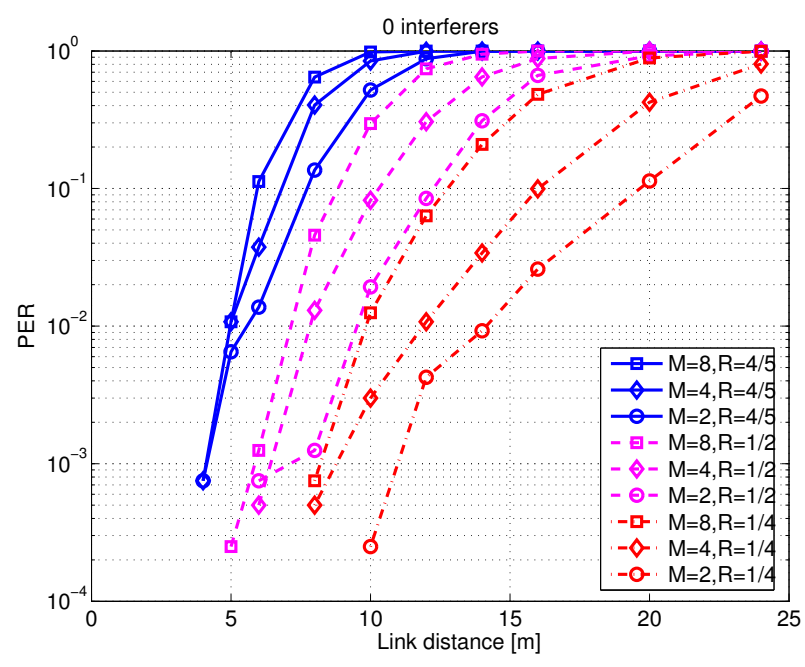

(a) PER with no MUI

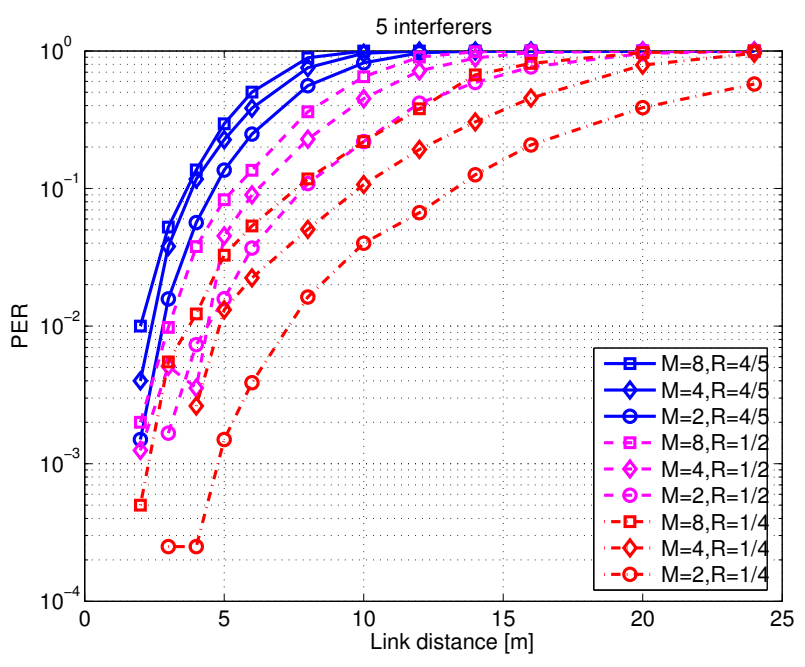

(b) PER with 5 interferers

Fig. 4. The curves correspond to the PER for $R_{c}=\frac{4}{5}, R_{c}=\frac{1}{2}$ and $R_{c}=\frac{1}{4}$ with $M=2,4,8$.

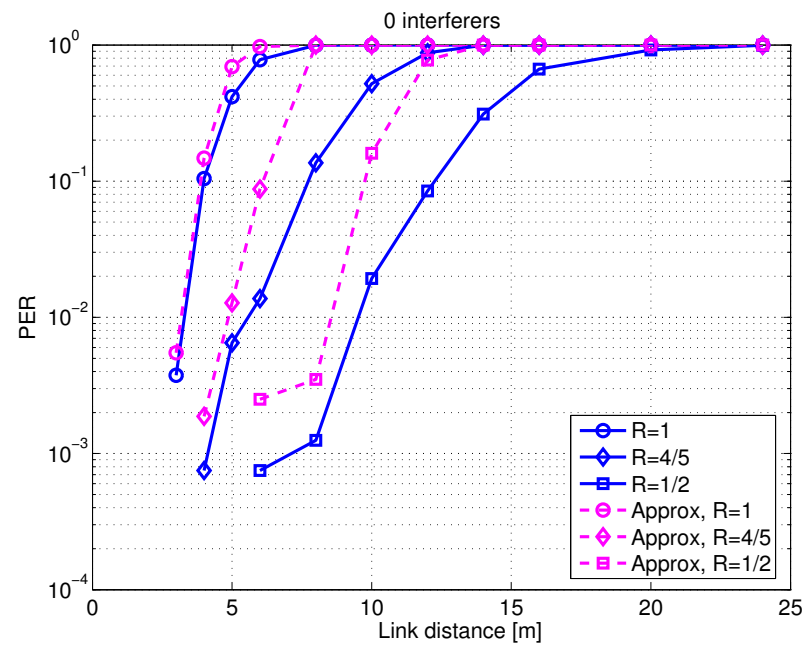

(a) $M=2$ and no interferers. With the channel code, the PER approximation is not valid.

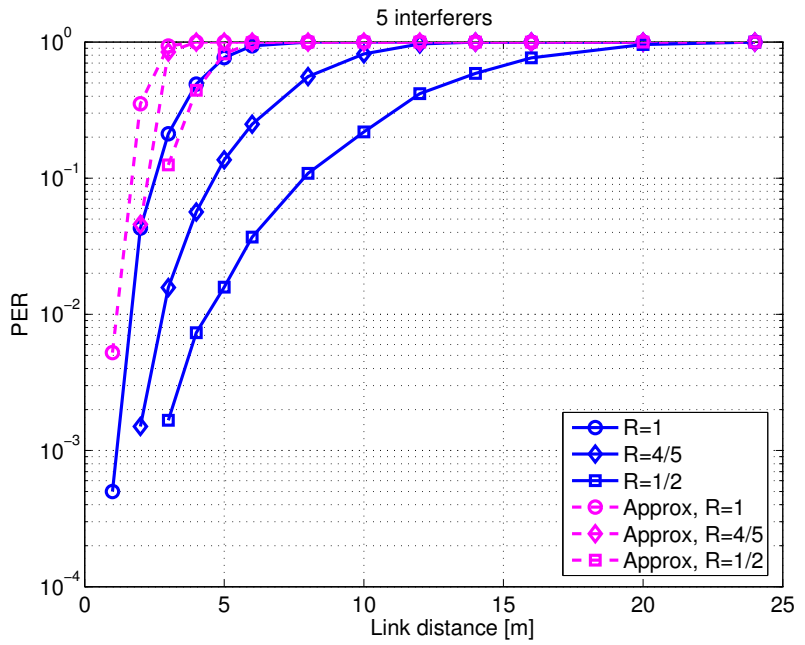

(b) $M=2$ and 5 interferers. Note how even in the uncoded case the PER approximation is not valid anymore due to the MUI.

Fig. 5. From left to right, the three plain curves correspond to the PER with no coding, $R_{c}=\frac{4}{5}$ and $R_{c}=\frac{1}{2}$ and $M=2$. The dashed curves are the PER approximation using the traditional approximation $1-(1-B E R)^{K}$. The approximation is valid only in the uncoded case without MUI.

combination of modulation and channel code are given in Table II. It is interesting to observe in Table II that every time the modulation order changes, the coding rate either decreases or stays constant. There are no coding rates augmentation when the modulation order decreases.

On Figure 6, we can clearly observe the rate decrease due to the MUI in near-far cases. When one interferer is present, the range is reduced by approximately 4 meters. For 5 interferers, it is reduced by 8 meters. The slope of the rate curve increases with the level of MUI. However, when the distance between the transmitter and the receiver is short relative to the distance between the interferers and the receiver, the MUI has practically no impact.

\section{A Note about the Power Consumption}

The energy per data bit $E_{d}$ is defined as $E_{d}=\frac{E_{P}}{k R_{c}}$ Hence $E_{d}$ decreases if either $k$ increases or the code rate $R_{c}$ increases. However, when $k$ is incremented, the number of matched filter outputs at the receiver is doubled. Hence, when $k$ is increased, the decrease of $E_{d}$ has to be compared with the 


\begin{tabular}{|c|c|c|c|c|c|c|c|c|c|c|c|}
\multicolumn{2}{|c|}{ Link distance in meters } & 1 & 2 & 3 & 4 & 5 & 6 & 8 & 10 & 12 & 14 \\
\hline \hline \multirow{2}{*}{$U=0$} & $M$ & 8 & 8 & 8 & 8 & 8 & 8 & 8 & 4 & 4 & 2 \\
\cline { 2 - 12 } & $R_{i d}$ & 0 & 0 & 2 & 2 & 4 & 6 & 12 & 16 & 22 & 24 \\
\hline \hline \multirow{2}{*}{$U=1$} & $M$ & 8 & 8 & 8 & 8 & 8 & 8 & 4 & 2 & n/a & n/a \\
\cline { 2 - 12 } & $R_{i d}$ & 0 & 0 & 2 & 4 & 12 & 12 & 20 & 24 & n/a & n/a \\
\hline \hline \multirow{2}{*}{$U=5$} & $M$ & 8 & 8 & 8 & 4 & 2 & 2 & n/a & n/a & n/a & n/a \\
\cline { 2 - 11 } & $R_{i d}$ & 0 & 2 & 8 & 8 & 12 & 16 & n/a & n/a & n/a & n/a \\
\hline
\end{tabular}

TABLE II

BEST COMBINATION OF MODULATION ORDER AND CHANNEL CODE

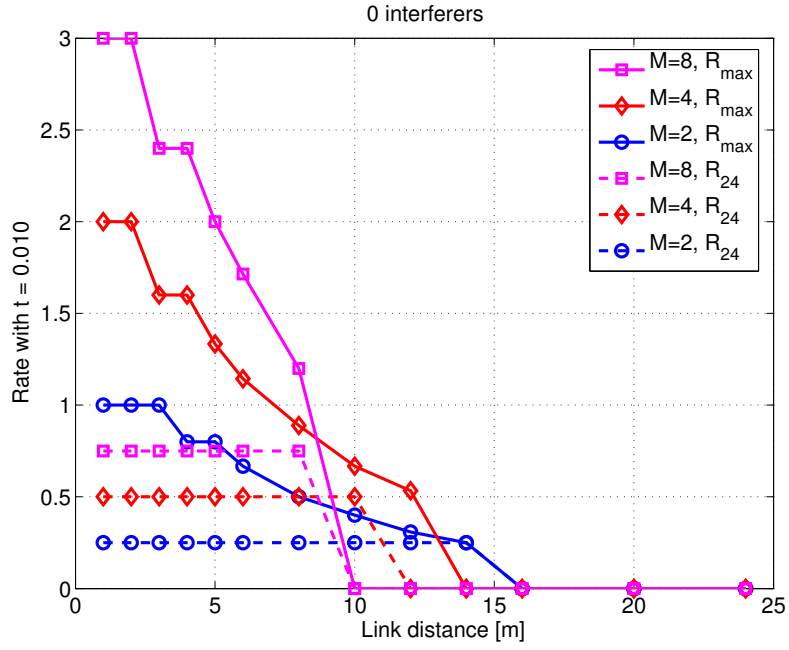

(a) Best rate versus link distance obtained for each modulation without multiuser interference.

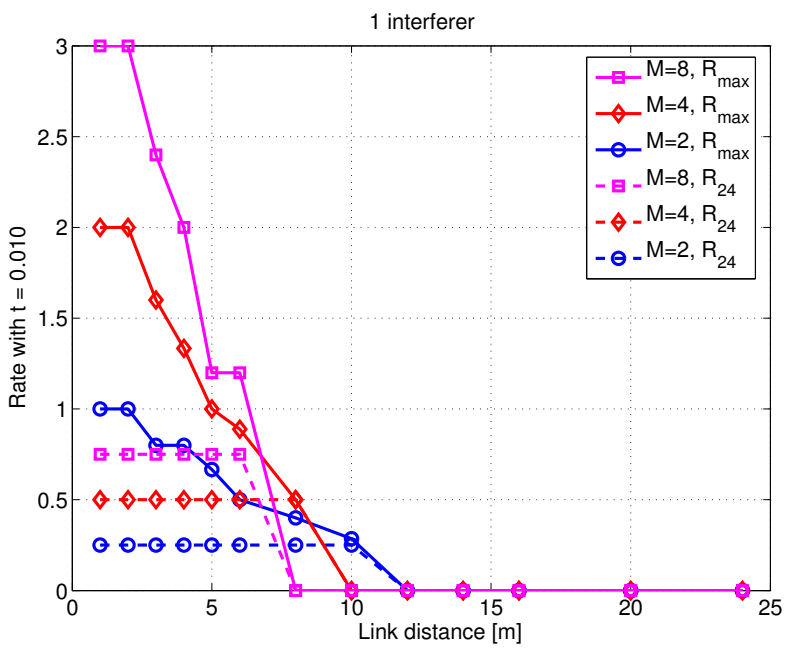

(b) Best rate versus link distance obtained for each modulation in the presence of 1 interferer at a distance of $1 \mathrm{~m}$ from the receiver.

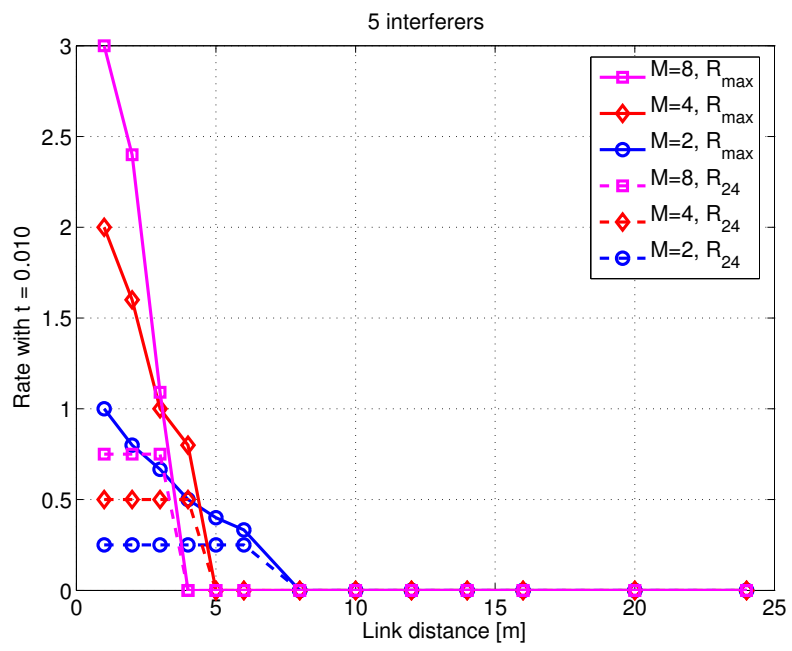

(c) Best rate versus link distance obtained for each modulation in the presence of 5 interferers at a distance of $1 \mathrm{~m}$ from the receiver.

Fig. 6. For various interference conditions, the plain curves represent the best rates obtained for each modulation order $M$ for $M=2,4,8$. For a given $M$ and link distance, the best rate is obtained by looking for the best channel code assuming a maximum PER. The dashed curves represent the rates obtained for each $M$ with the channel code of lowest rate. These figure clearly show the benefit of being able to adapt the code rate and the modulation in various interference conditions, even in the presence of strong near-far interference. 
energy required to produce the $M=2^{k}$ matched filters.

\section{CONCLUSION}

BIC M-ary PPM appears to be perfectly suitable for an adaptive system as in [1], [2]. In the case of a channel without multiuser interference, the modulation could be easily adapted to the channel condition. Indeed, the receiver could track one or several channel metrics such as the bit metrics at the output of the decoder (see (14)), the received energy, or the M-ary PPM channel capacity. However, in the case of a channel with interfering users, the choice of an appropriate metric and adaptation algorithm remains open.

It would be interesting to study the performance of BIC M-ary PPM with an iterative decoder as is done with BICMID [18] as well as the performance with an energy detector instead of a Rake receiver. Also, in the presence of nearfar interference, an interference mitigation [2] scheme could increase the performance.

\section{REFERENCES}

[1] J. Y. Le Boudec, R. Merz, B. Radunovic, and J. Widmer, "DCC-MAC: A decentralized MAC protocol for 802.15.4a-like UWB mobile adhoc networks based on dynamic channel coding," in Proceedings of Broadnets, San Jose, October 2004.

[2] R. Merz, J. Widmer, J.-Y. Le Boudec, and B. Radunovic, "A joint PHY/MAC architecture for low-radiated power TH-UWB wireless adhoc networks," Wireless Communications and Mobile Computing Journal, Special Issue on Ultrawideband (UWB) Communications, vol. 5, no. 5, pp. 567-580, August 2005.

[3] G. Caire, G. Taricco, and E. Biglieri, "Bit-interleaved coded modulation," IEEE Transactions on Information Theory, vol. 44, no. 3, pp. 927-946, May 1998.

[4] E. Zehavi, "8-PSK trellis codes for a rayleigh channel," IEEE Transactions on Communications, vol. 40, no. 5, pp. 873-884, May 1992.

[5] F. Ramirez-Mireles, "Performance of ultrawideband SSMA using time hopping and m-ary PPM," IEEE Journal on Selected Areas in Communications, vol. 19, no. 6, pp. 1186-1196, June 2001.

[6] A. Forouzan and M. Abtahi, "Application of convolutional error correcting codes in ultrawideband m-ary PPM signaling," IEEE Microwave and Wireless Components Letters, vol. 13, no. 8, pp. 308-310, August 2003.

[7] K. Takizawa and R. Kohno, "Combined iterative demapping and decoding for coded UWB-IR systems," in IEEE Conference on Ultra Wideband Systems and Technologies, November 2003, pp. 423-427.
[8] - "Combined iterative demapping and decoding for coded m-ary BOK DS-UWB systems," in Joint IEEE Conference on Ultra Wideband Systems and Technologies \& IWUWBS, May 2004, pp. 207-211.

[9] S. Yoshida and I. Ohtsuki, "Performance evaluation of adaptive internally turbo coded ultra wideband-impulse radio (aitc-uwb-ir) in multipath channels," in IEEE 60th Vehicular Technology Conference, vol. 2, September 2004, pp. 1179-1183.

[10] N. August, R. Thirugnanam, and D. Ha, "An adaptive UWB modulation scheme for optimization of energy, BER, and data rate," in UWBST, 2004, pp. 182-186.

[11] I. Guvenc, H. Arslan, S. Gezici, and H. Kobayashi, "Adaptation of multiple access parameters in time hopping UWB cluster based wireless sensor networks," in IEEE International Conference on Mobile Ad-hoc and Sensor Systems (MASS), October 2004, pp. 235-244.

[12] R. Merz and J.-Y. Le Boudec, "Conditional bit error rate for an impulse radio UWB channel with interfering users," in IEEE International Conference on Ultrawideband (ICU 2005), September 2005.

[13] J. Hagenauer, "Rate-compatible punctured convolutional codes (RCPC codes) and their applications," IEEE Transactions on Communications, vol. 36, no. 4, pp. 389-400, April 1988.

[14] M. Win, G. Chrisikos, and N. Sollenberger, "Effects of chip rate on selective RAKE combining," IEEE Communications Letters, vol. 4, no. 7, pp. 233-235, July 2000.

[15] P. Frenger, P. Orten, T. Ottosson, and A. Svensson, "Rate-compatible convolutional codes for multirate DS-CDMA systems," IEEE Transactions on Communications, vol. 47, no. 6, pp. 828-836, June 1999.

[16] A. F. Molisch, K. Balakrishnan, C.-C. Chong, S. Emami, A. Fort, J. Karedal, J. Kunisch, H. Schantz, U. Schuster, and K. Siwiak, "IEEE 802.15.4a channel model - final report, document 04/662r0," Available at http://www.ieee802.org/15/pub/TG4a.html, November 2004.

[17] S. Ghassemzadeh, R. Jana, C. Rice, W. Turin, and V. Tarokh, "Measurement and modeling of an ultra-wide bandwidth indoor channel," IEEE Transactions on Communications, vol. 52, no. 10, pp. 1786-1796, October 2004

[18] X. Li, A. Chindapol, and J. Ritcey, "Bit-interleaved coded modulation with iterative decoding and 8 PSK signaling," IEEE Transactions on Communications, vol. 50, no. 8, pp. 1250-1257, August 2002. 